\title{
Clinical Value of Fecal Calprotectin in Predicting Mucosal Healing in Patients With Ulcerative Colitis
}

\author{
Fang Chen ${ }^{1}$, Yue $\mathrm{Hu}^{2}$, Yi-Hong Fan ${ }^{2 *}$ and Bin $\mathrm{Lv}^{2}$ \\ ${ }^{1}$ Department of Gastroenterology, Zhejiang Hospital of Integrated Traditional Chinese and Western Medicine, Hangzhou, \\ China, ${ }^{2}$ Department of Gastroenterology, The First Affiliated Hospital of Zhejiang Chinese Medical University, Hangzhou, \\ China
}

OPEN ACCESS

Edited by:

Xiang Xue,

University of New Mexico,

United States

Reviewed by:

Giulia Roda,

Humanitas University, Italy

Tsvetelina Velikova,

Lozenetz Hospital, Bulgaria

*Correspondence:

Yi-Hong Fan

yhfansjr@163.com

Specialty section:

This article was submitted to

Gastroenterology,

a section of the journal

Frontiers in Medicine

Received: 11 March 2021

Accepted: 07 June 2021

Published: 03 August 2021

Citation:

Chen F, Hu Y, Fan Y-H and LV B

(2021) Clinical Value of Fecal Calprotectin in Predicting Mucosal Healing in Patients With Ulcerative

Colitis. Front. Med. 8:679264.

doi: 10.3389/fmed.2021.679264
Aim: This study aimed to evaluate the clinical significance of fecal calprotectin (FC) in assessment of ulcerative colitis (UC) patients' endoscopic patterns and clinical manifestation.

Methods: A total of 143 UC patients who received colonoscopy and 108 controls were included. After providing stool samples, patients underwent total colonoscopy. FC was measured by an enzyme-linked immunosorbent assay (ELISA). Clinical activity was based on the Mayo score. Endoscopic findings was scored by the Ulcerative Colitis Endoscopic Index of Severity (UCEIS). Correlation analysis and receiver-operator characteristic (ROC) analysis were carried out to determine the significance of measurements.

Results: The median (interquartile range, IQR) of FC levels was 211 (43-990) $\mu \mathrm{g} / \mathrm{g}$ in UC and $87.5(40.50 \sim 181) \mu \mathrm{g} / \mathrm{g}$ in the control group. Fecal calprotectin correlated significantly with both Mayo and UCEIS scores (Spearman's r 0.670 and $0.592, P<$ 0.01). With a cut-off value of $164 \mu \mathrm{g} / \mathrm{g}$ for fecal calprotectin concentration, the area under the curve $(A \cup C)$ in receiver operator characteristic analysis was 0.830 , sensitivity was $85.42 \%$, specificity was $73.68 \%$, positive predictive value (PPV) was $62.12 \%$, and negative predictive value (NPV) was $9.10 \%$ in predicting clinical active disease. Similarly, the power of FC to predict mucosal healing $(\mathrm{MH})$ was modest. With a cut-off value of $154.5 \mu \mathrm{g} / \mathrm{g}$, the AUC was 0.839 , sensitivity was $72.34 \%$, and specificity was $85.71 \%$.

Conclusion: For evaluating the disease activity of UC, FC is a clinically relevant biomarker for both clinically active disease and $\mathrm{MH}$ in patients with UC. But the cut-off value still needs large and multicenter studies for confirmation.

Keywords: biomarkers, ulcerative colitis, fecal calprotectin, mucosal healing, clinical value

\section{INTRODUCTION}

Ulcerative colitis (UC) is a chronic disease with a remitting and relapsing course. For evaluation of disease course and for monitoring treatment response, reliable tools are essential. Assessment of UC activity in clinic is usually based on a combination of clinical manifestations and laboratory tests. The current gold standard is colonoscopy because symptoms do not precisely reflect intestinal inflammation and mucosal healing (1). Endoscopic procedures, however, are unpleasant, sometimes painful, and time-consuming in China. Fecal calprotectin (FC) is a calciumbinding, cytosolic protein in neutrophils which has antimicrobial and antiproliferative properties. 
Fecal calprotectin concentration reflects the increased migration of neutrophils through the inflamed bowel wall to the lumen (2). In stool, calprotectin is degradation-resistant, stable, and easily measurable by ELISA (3). The test has been used successfully to distinguish inflammatory from functional bowel disorders (4). Recent studies suggested that FC levels correlate well with endoscopic indices of UC activity including Matts' index (5), Sutherland criteria (6), Rachmilewitz index (7), and the Mayo endoscopic subscore (8). In addition, elevated FC may indicate an increased risk of disease relapse $(9,10)$.

Since longstanding active inflammation is also considered a risk factor for the development of tissue destruction, dysplasia, and cancer (11), healing of the mucosa may also lead to a reduction in those complications. For these reasons, mucosal healing has been brought into the treat to target era. The current study found that a subgroup of patients had persistently active endoscopic inflammation while in clinical remission (12). Obviously, a noninvasive biomarker to identify patients with $\mathrm{MH}$ is preferable in clinical settings. This could allow more regular assessment of inflammation and possibly lead to a reduced requirement for follow-up endoscopies.

In recent years, various biomarkers of $\mathrm{MH}$ have been explored such as C-reactive protein (CRP) and erythrocyte sedimentation rate (ESR). Because in UC patients, inflammation is mainly confined to the colon and the rectum, it may be reasonable that a fecal marker is more accurate than a serum marker.

The aim of this study was to evaluate the clinical significance of FC in the assessment of UC clinical activity and $\mathrm{MH}$. Additionally, cut-off levels were also determined for the clinical activity and $\mathrm{MH}$.

\section{METHODS}

\section{Patients}

A total of 143 adult outpatients and inpatients with a previously confirmed diagnosis of UC referred for colonoscopy at the Departments of Gastroenterology of the First Affiliated Hospital of Zhejiang Chinese Medical University between May 2015 and December 2016 were included. They were diagnosed on the basis of clinical, endoscopic, and histologic criteria. A second cohort of 108 healthy volunteers served as controls. The disease extension was classified according to the Montreal classification (13). Exclusion criteria included pregnancy, colorectal cancer, history of bowel resection, long-term use of NSAIDs, or presence of comorbidities that could cause inflammatory reactions, active infection, incomplete colonoscopy (not reaching the cecum), and inability to provide stool samples.

Clinical disease severity was assessed according to Mayo scores. Clinical disease activity was divided into clinical remission (0-2), mild (3-5), moderate (6-10), and severe (11-12) according to the frequency of defecation, hematochezia, and findings of colonoscopy and physician's global assessment. The UCEIS score $(14,15)$, composed of vascular pattern $(0-2)$, bleeding $(0-3)$, and erosions and ulcers (0-3), was applied to evaluate endoscopic activity, while MH (16) was defined as UCEIS 0 or 1, and UCEIS 1 was limited to vascular patterns.

\section{Study Protocol}

Patients provided stool samples within the previous 7 days of the colonoscopy (prior to bowel preparation), and the stool samples were stored at $-20^{\circ} \mathrm{C}$ until assay. After bowel preparation, patients underwent total colonoscopy, and UCEIS score was used to assess $\mathrm{MH}$. The greatest score in any anatomical site was recorded.

\section{Fecal Calprotectin Assays}

Stools were collected within the previous 7 days of the colonoscopy, and immediately stored at $-20^{\circ} \mathrm{C}$. The stool samples were sent to Suzhou Herui IBD Project Center, and fecal calprotectin was measured in a blind manner using the PhiCal enzyme-linked immunosorbent assay (ELISA) Assay.

\section{Statistics}

For numerical variables, median and interquartile range (IQR) were calculated, and the Mann-Whitney $U$-test was applied. The Spearman correlation analysis between FC and clinical/endoscopic disease severity was carried out. The best cutoff for FC to predict clinical activity and $\mathrm{MH}$ were calculated by using receiver-operator characteristic (ROC) graphs. According to the cut-off levels, test significance including sensitivity (SENS), specificity (SPEC), positive-predictive value (PPV), negative predictive value (NPV), and accuracy rate (AR) were calculated. Two sided $P<0.05$ were considered to be statistically significant.

\section{RESULTS}

\section{Characteristics of the Participants}

Overall, 143 UC patients and 108 controls were included in the study. Among the 143 UC patients (44\% women), the mean age at the time of inclusion was $43.64 \pm 13.62$ years. While ulcerative colitis extent was limited to the rectum in 52

\begin{tabular}{|c|c|c|}
\hline & UC & Control \\
\hline $\mathrm{N}$ & 143 & 108 \\
\hline Male/female & $80 / 60$ & $47 / 61$ \\
\hline Age (Mean \pm SD) & $43.64 \pm 13.62$ & $48.53 \pm 16.30$ \\
\hline \multicolumn{3}{|l|}{ Age at diagnosis (years) } \\
\hline $\mathrm{A} 1(\leq 16)$ & 0 & \\
\hline A2 (17-40) & 88 (61.54\%) & \\
\hline$A 3(\geq 40)$ & 55 (38.46\%) & \\
\hline \multicolumn{3}{|l|}{ Disease location } \\
\hline Non & 20 (13.99\%) & \\
\hline E1 & 52 (36.36\%) & \\
\hline E2 & 27 (18.88\%) & \\
\hline E3 & 44 (30.77\%) & \\
\hline \multicolumn{3}{|l|}{ Mayo grades } \\
\hline Remission $(\leq 2)$ & 49 (34.27\%) & \\
\hline Mild activity (3-5) & $46(32.17 \%)$ & \\
\hline Moderate activity (6-10) & $41(28.67 \%)$ & \\
\hline Severe activity (11-12) & 7 (4.90\%) & \\
\hline
\end{tabular}


TABLE 2 | Median fecal calprotectin levels (interquartile range) in patients stratified according to the Mayo grades ( $\mu \mathrm{g} / \mathrm{g}$ ).

\begin{tabular}{|c|c|c|c|c|c|}
\hline \multirow[t]{2}{*}{ Variable } & \multirow[t]{2}{*}{$\mathbf{N}$} & \multicolumn{4}{|c|}{ FC $(\mu g / g)$} \\
\hline & & Median & Quartile & Min Max & Interquartile range (IQR) \\
\hline Control & 108 & 87.5 & 141 & $11 \sim 1,560$ & $40.50 \sim 181$ \\
\hline UC & 143 & 211 & 947 & $17 \sim 6,964$ & 43 990 \\
\hline Remission & 49 & $38^{\star}$ & 73 & $22 \sim 5,321$ & $30 \sim 102.5$ \\
\hline Mild activity & 46 & 220.5 & 281 & $17 \sim 5,235$ & $87 \sim 367.75$ \\
\hline Moderate activity & 41 & $1,138 \Delta$ & 2359 & $26 \sim 6,964$ & $340.50 \sim 2,699$ \\
\hline Severe activity & 7 & $2,481 \Delta \Delta$ & 2494 & $1,414 \sim 6,324$ & $1,573 \sim 4,067$ \\
\hline
\end{tabular}

${ }^{*} p<0.05$ ( $\left.p=0.002\right)$, vs. the control; $\Delta p<0.05$ ( $\left.p=0.000\right)$, vs. the mild group; $\Delta \Delta p<0.05(p=0.000, p=0.033$, respectively), vs. the mild and moderate group.

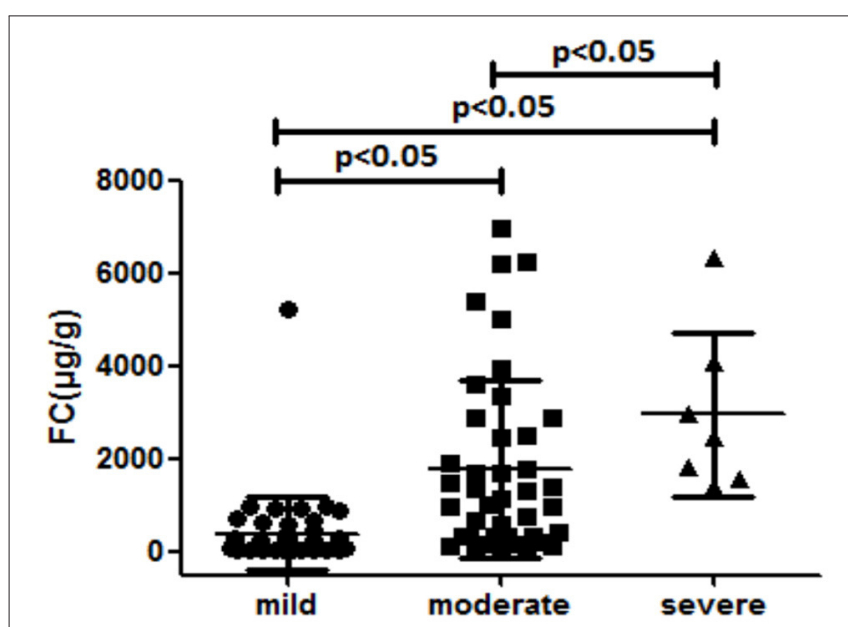

FIGURE 1 | Median fecal calprotectin levels (interquartile range) in patients stratified according to the Mayo grades $(\mu \mathrm{g} / \mathrm{g})$.

patients (36.36\%), 27 patients (18.88\%) had sigmoid/left colon involvement and 44 patients $(30.77 \%)$ had pancolitis. Patients' characteristics are shown in Table 1. According to Mayo scores, $49(34.27 \%)$ patients were in remission, 46 (32.17\%) patients had mild, 41 (28.67\%) patients had moderate, and 7 (4.90\%) patients had severe disease activity. Overall, mucosal healing, defined as UCEIS score 0 or 1 , was observed in 48 ulcerative colitis patients (33.57\%).

In total, 108 controls were studied (56\% women). Their median age was $48.53 \pm 16.30$ years. The median fecal calprotectin in this group was 87.5 (IQR 40.50-181) $\mu \mathrm{g} / \mathrm{g}$. The median (IQR) value for FC level of all patients was 211 (43-990) $\mu \mathrm{g} / \mathrm{g}$. There was a significant difference in the FC concentration between the UC and the controls $(P<0.05$; Table 2). The FC concentration were 38 (30-102.5) $\mu \mathrm{g} / \mathrm{g}, 220.5$ (87-367.75) $\mu \mathrm{g} / \mathrm{g}, 1,138$ (340.50-2699) $\mu \mathrm{g} / \mathrm{g}$, and 2,481 (1573-4067) $\mu \mathrm{g} / \mathrm{g}$, respectively with each stage classified by Mayo scores. As seen in Figure 1 and Table 2, there was a significant difference in FC levels between patients with mild disease and moderate disease $(P<0.05)$ as well as between moderate disease and severe disease $(P<0.05)$.

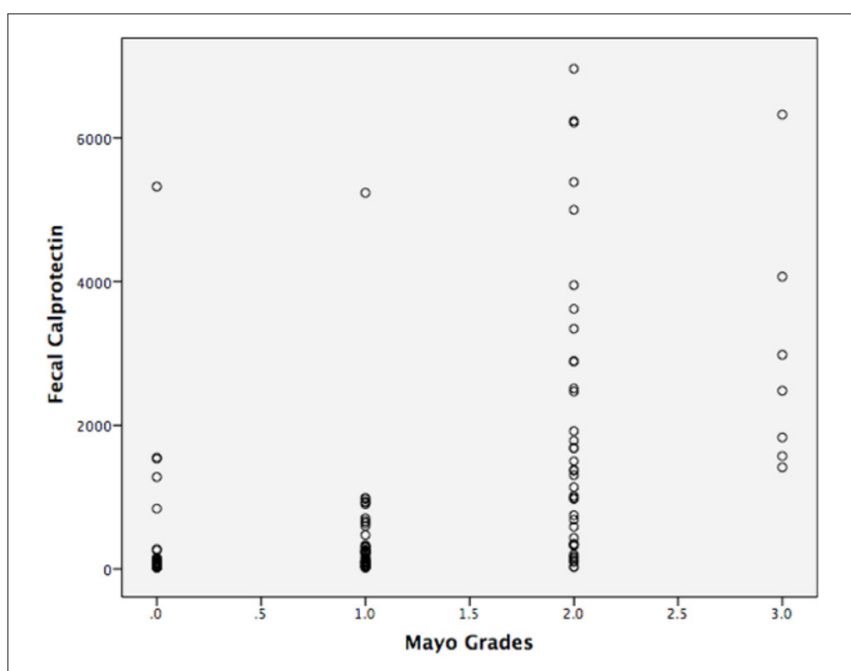

FIGURE 2 | Concentrations of fecal calprotectin and the Mayo grades of UC $(r$ $=0.670, p<0.01$ ).

\section{Correlation Analysis}

The correlation analysis is shown in Figures 2, 3. The Mayo grades and the UCEIS scores both correlated very well with the FC levels $(r=0.670, P<0.01$, and $r=0.592, P<$ 0.01 , respectively).

\section{ROC Curve Analysis}

Using a ROC curve, we attempted to determine the best cutoff value of FC to detect clinical activity and $\mathrm{MH}$. The area under the ROC curve to predict clinical activity and $\mathrm{MH}$ was 0.830 and 0.839 , respectively (Figures 4, 5). The best cut-off point to detect clinical activity was $164 \mu \mathrm{g} / \mathrm{g}$ (sensitivity $85.42 \%$, specificity 73.68\%, PPV 62.12\%, NPV 9.10\%, AR 77.62\%). A cutoff value of $154.5 \mu \mathrm{g} / \mathrm{g}$ indicated $\mathrm{MH}$, with sensitivity of $72.34 \%$, specificity of $85.71 \%$, PPV $90.67 \%$, NPV $38.24 \%$, and AR $76.92 \%$.

\section{DISCUSSION}

In the present study, we assessed the correlation between fecal calprotectin level and clinical/endoscopic scores in UC and 

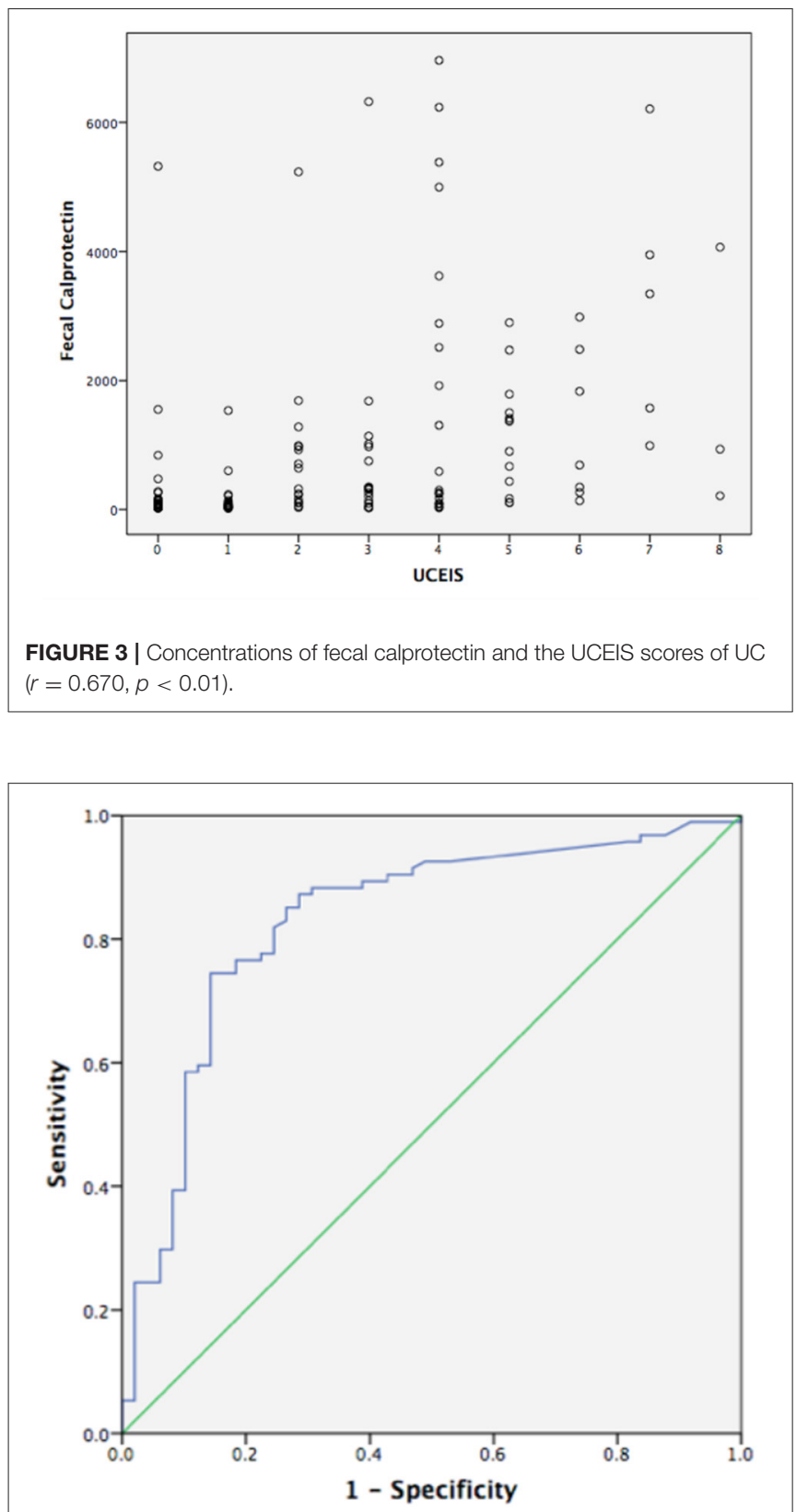

FIGURE 4 | Receiver operating curve analysis (ROC) of fecal calprotectin in detecting clinical activity (AUC 0.830, $p<0.05,95 \% \mathrm{Cl} 0.755 \sim 0.904$ ).

showed the performances of FC in detecting clinical activity and endoscopic mucosal healing.

Fecal calprotectin is an abundant protein in neutrophils, which infiltrates the mucosa during inflammation. Data support its use in differentiating inflammatory bowel disease (IBD) from irritable bowel syndrome (IBS) (17-20), evaluating abdominal discomfort (21). Several reports have shown that FC level correlates well with clinical, endoscopic, and histological parameters of disease activity $(6,19)$ in UC patients. To some extent, FC may reflect disease activity in UC better than in CD

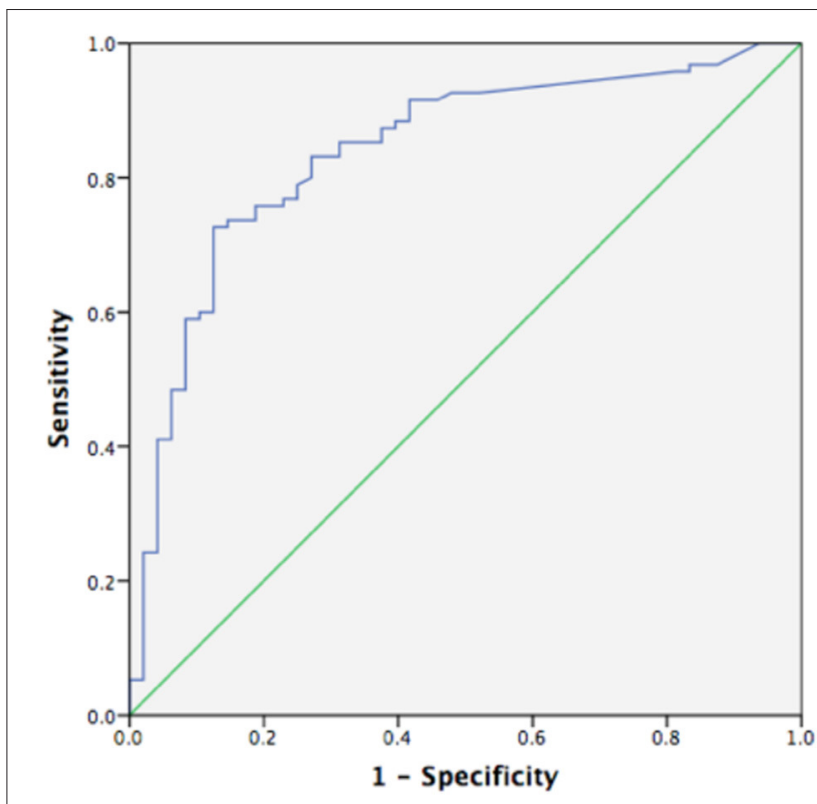

FIGURE 5 | Receiver operating curve analysis (ROC) of fecal calprotectin in $\mathrm{MH}$ (AUC 0.839, $p<0.05,95 \% \mathrm{Cl} 0.769 \sim 0.909$ ).

as some authors reported (22). FC determination may also be useful in predicting impending clinical relapse especially during the following 3 months in both CD and UC patients (23). FC is also useful in assessing treatment response (24-26).

In the management of patients with UC, endoscopy has an essential role in viewing and evaluating the severity of disease activity in the intestinal mucosa as well as assessing the efficacy of treatment modalities. However, discordance in clinical manifestations and endoscopic findings is not rare. Clinical indices are not reliable in assessing endoscopic $\mathrm{MH}$ and in predicting the disease course $(27,28)$. Evolving evidence indicates that $\mathrm{MH}$ is associated with lower risk of longterm complications (29-31). Therefore, currently, $\mathrm{MH}$ is of great interest to gastroenterologists and considered as an ideal therapeutic target. However, the exact definition of $\mathrm{MH}$ continues to be controversial and several scoring systems have been developed. In our study, we applied UCEIS to define MH as the remission stratum that corresponds to UCEIS 0 or 1. Further, we limited the UCEIS score 1 to a vascular pattern descriptor, so that score 1 of the bleeding descriptor and score 1 of the erosions and ulcers descriptor do not mean real MH. Arai et al. (32) recently reported that UCEIS is useful to predict clinical outcomes and long-term prognosis in UC patients with clinical remission. Consequently, FC had a good correlation with UCEIS. Additionally, we suggest that a definition of $\mathrm{MH}$ based on the UCEIS scores may be more relevant.

A recent systematic review (33) showed that fecal markers like FC are promising non-invasive indicators of $\mathrm{MH}$. It is imperative that non-invasive markers become available for routine clinical use. In other words, this could allow more regular assessment of inflammation with subsequent timely clinical 
decisions and possibly lead to a reduced requirement for followup endoscopies. Schoepfer's study (7), the largest study so far, described the diagnostic efficiency of FC to predict mucosal inflammation with sensitivity 93\%, specificity 71\%, PPV 91\%, and NPV $81 \%$ using a cut-off $50 \mu \mathrm{g} / \mathrm{g}$. Yamaguchi et al. (34) analyzed the correlation between FC with both Mayo endoscopic subscore 0 or Mayo endoscopic subscore 0 and 1 defining $\mathrm{MH}$. Not surprisingly, specificity and PPV were greater when using the Mayo 0 score. Based on the interpretations of the ROC graphs, using UCEIS defining $\mathrm{MH}$, we obtained a cut-off FC level of $154.5 \mu \mathrm{g} / \mathrm{g}$ to predict $\mathrm{MH}$ with sensitivity $72.34 \%$, specificity $85.71 \%$, and PPV $90.67 \%$. It is not surprising that there has been no agreement regarding an appropriate cut-off level for FC to predict $\mathrm{MH}$ (35). Our results are reasonably comparable with these previously published data.

Our sample size could be considered as a limitation of our study. Second, using FC as a predictive tool for $\mathrm{MH}$ requires analysis from clinically quiescent patients. This is the biggest weaknesses in our study. Third, the FC levels have also been shown to be variable (36), to overcome this problem we ensured that all patients provided stool samples at least 1 week post biologic administrations. Combination of clinical symptoms and serum and fecal biomarkers is likely to be superior to one single parameter. Such analyses will require well-powered and multicenter studies.

In conclusion, fecal calprotectin could reflect the disease activity of UC and are rational fecal markers of intestinal inflammation for clinical application. FC is also a clinically relevant biomarker of $\mathrm{MH}$ in patients with $\mathrm{UC}$, but the value of the cut-off still needs large and multicenter studies for confirmation.

\section{REFERENCES}

1. Gomes P, du Boulay C, Smith CL, Holdstock G. Relationship between disease activity indices and colonoscopic findings in patients with colonic inflammatory bowel disease. Gut. (1986) 27:92-5. doi: 10.1136/gut.27.1.92

2. Røseth AG, Schmidt PN, Fagerhol MK. Correlation between faecal excretion of indium-111-labelled granulocytes and calprotectin, a granulocyte marker protein, in patients with inflammatory bowel disease. Scand J Gastroenterol. (1999) 34:50-4. doi: 10.1080/00365529950172835

3. Røseth AG, Fagerhol MK, Aadland E, Schjønsby H. Assessment of the neutrophil dominating protein calprotectin in feces. A methodologic study. Scand J Gastroenterol. (1992) 27:793-8. doi: 10.3109/00365529209011186

4. Carroccio A, Iacono G, Cottone M, Di Prima L, Cartabellotta F, Cavataio F et al. Diagnostic accuracy of fecal calprotectin assay in distinguishing organic causes of chronic diarrhea from irritable bowel syndrome: a prospective study in adults and children. Clin Chem. (2003) 49(6 Pt 1):8617. doi: $10.1373 / 49.6 .861$

5. Hanai H, Takeuchi K, Iida T, Kashiwagi N, Saniabadi AR, Matsushita I et al. Relationship between fecal calprotectin, intestinal inflammation, and peripheral blood neutrophils in patients with active ulcerative colitis. Dig Dis Sci. (2004) 49:1438-43. doi: 10.1023/B:DDAS.0000042243.47279.87

6. Xiang JY, Ouyang Q, Li GD, Xiao NP. Clinical value of fecal calprotectin in determining disease activity of ulcerative colitis. World J Gastroenterol. (2008) 14:53-7. doi: 10.3748/wjg.14.53

7. Schoepfer AM, Beglinger C, Straumann A, Trummler M, Renzulli P, Seibold F. Ulcerative colitis: correlation of the Rachmilewitz endoscopic activity index with fecal calprotectin, clinical activity, C-reactive protein, and blood leukocytes. Inflamm Bowel Dis. (2009) 15:1851-8. doi: 10.1002/ibd.20986

\section{DATA AVAILABILITY STATEMENT}

The raw data supporting the conclusions of this article will be made available by the authors, without undue reservation.

\section{ETHICS STATEMENT}

The studies involving human participants were reviewed and approved by Ethics Committee of the First Affiliated Hospital of Zhejiang University of Traditional Chinese Medicine. The patients/participants provided their written informed consent to participate in this study.

\section{AUTHOR CONTRIBUTIONS}

Y-HF designed the report. FC and $\mathrm{YH}$ collected the clinical data. BL contributed to revising the manuscript. FC wrote the manuscript. All authors contributed to the article and approved the submitted version.

\section{FUNDING}

Supported by the National Natural Science Foundation of China, No. 81473506; the Ministy of Construction, Zhejiang Province, No. WKJ-ZJ-1531; the National Natural Science Foundation of Zhejiang Province, No. LY17H290009; the State Administration of Traditional Chinese Medicine of Zhejiang Province, Nos. $2016 Z B 047$ and 2017ZA056; the Medical and Health Care Foundation of Zhejiang Province Science, No. 2015RCA021.

8. D'Haens G, Ferrante M, Vermeire S, Baert F, Noman M, Moortgat L et al. Fecal calprotectin is a surrogate marker for endoscopic lesions in inflammatory bowel disease. Inflamm Bowel Dis. (2012) 18:2218-24. doi: 10.1002/ibd.22917

9. Tibble JA, Sigthorsson G, Bridger S, Fagerhol MK, Bjarnason I. Surrogate markers of intestinal inflammation are predictive of relapse in patients with inflammatory bowel disease. Gastroenterology. (2000) 119:15-22. doi: 10.1053/gast.2000.8523

10. Maiden L, Takeuchi K, Baur R, Bjarnason I, O’Donohue J, Forgacs I et al. Selective white cell apheresis reduces relapse rates in patients with IBD at significant risk of clinical relapse. Inflamm Bowel Dis. (2008) 14:14138. doi: 10.1002/ibd.20505

11. Rutter M, Saunders B, Wilkinson K, Rumbles S, Schofield G, Kamm M et al. Severity of inflammation is a risk factor for colorectal neoplasia in ulcerative colitis. Gastroenterology. (2004) 126:451-9. doi: 10.1053/j.gastro.2003. 11.010

12. Rosenberg L, Lawlor GO, Zenlea T, Goldsmith JD, Gifford A, Falchuk $\mathrm{KR}$ et al. Predictors of endoscopic inflammation in patients with ulcerative colitis in clinical remission. Inflamm Bowel Dis. (2013) 19:77984. doi: 10.1097/MIB.0b013e3182802b0e

13. Satsangi J, Silverberg MS, Vermeire S, Colombel JF. The montreal classification of inflammatory bowel disease: controversies, consensus, and implications. Gut. (2006) 55:749-53. doi: 10.1136/gut.2005.082909

14. Travis SP, Schnell D, Krzeski P, Abreu MT, Altman DG, Colombel JF et al. Developing an instrument to assess the endoscopic severity of ulcerative colitis: the ulcerative colitis endoscopic index of severity (UCEIS). Gut. (2012) 61:535-42. doi: 10.1136/gutjnl-2011-300486

15. Travis SP, Schnell D, Krzeski P, Abreu MT, Altman DG, Colombel $\mathrm{JF}$ et al. Reliability and initial validation of the ulcerative 
colitis endoscopic index of severity. Gastroenterology. 145:987-95. doi: 10.1053/j.gastro.2013.07.024

16. Ikeya K, Hanai H, Sugimoto K, Osawa S, Kawasaki S, Iida $\mathrm{T}$ et al. The ulcerative colitis endoscopic index of severity more accurately reflects clinical outcomes and long-term prognosis than the mayo endoscopic score. J Crohns Colitis. (2016) 10:286-95. doi: 10.1093/ecco-jcc/jjv210

17. Sutherland AD, Gearry RB, Frizelle FA. Review of fecal biomarkers in inflammatory bowel disease. Dis Colon Rectum. (2008) 51:128391. doi: 10.1007/s10350-008-9310-8

18. Schoepfer AM, Trummler M, Seeholzer P, Seibold-Schmid B, Seibold F. Discriminating IBD from IBS: comparison of the test performance of fecal markers, blood leukocytes, CRP, and IBD antibodies. Inflamm Bowel Dis. (2008) 14:32-9. doi: 10.1002/ibd.20275

19. Gisbert JP, McNicholl AG. Questions and answers on the role of faecal calprotectin as a biological marker in inflammatory bowel disease. Dig Liver Dis. (2009) 41:56-66. doi: 10.1016/j.dld.2008.05.008

20. Mumolo MG, Bertani L, Ceccarelli L, Laino G, Di Fluri G, Albano E et al. From bench to bedside: fecal calprotectin in inflammatory bowel diseases clinical setting. World J Gastroenterol. (2018) 24:368194. doi: $10.3748 /$ wjg.v24.i33.3681

21. Manz M, Burri E, Rothen C, Tchanguizi N, Niederberger C, Rossi L et al. Value of fecal calprotectin in the evaluation of patients with abdominal discomfort: an observational study. BMC Gastroenterol. (2012) 12:5. doi: 10.1186/1471-230X-12-5

22. Costa F, Mumolo MG, Ceccarelli L, Bellini M, Romano MR, Sterpi C et al. Calprotectin is a stronger predictive marker of relapse in ulcerative colitis than in Crohn's disease. Gut. (2005) 54:364-8. doi: 10.1136/gut.2004.0 43406

23. Gisbert JP, Bermejo F, Pérez-Calle JL, Taxonera C, Vera I, McNicholl AG et al. Fecal calprotectin and lactoferrin for the prediction of inflammatory bowel disease relapse. Inflamm Bowel Dis. (2009) 15:1190-8. doi: 10.1002/ibd. 20933

24. Wagner M, Peterson CG, Ridefelt P, Sangfelt P, Carlson M. Fecal markers of inflammation used as surrogate markers for treatment outcome in relapsing inflammatory bowel disease. World J Gastroenterol. (2008) 14:55849; discussion 5588. doi: 10.3748/wjg.14.5584

25. Sipponen T, Björkesten CG, Färkkilä M, Nuutinen H, Savilahti E, Kolho KL. Faecal calprotectin and lactoferrin are reliable surrogate markers of endoscopic response during Crohn's disease treatment. Scand J Gastroenterol. (2010) 45:325-31. doi: 10.3109/00365520903483650

26. Langhorst J, Elsenbruch S, Koelzer J, Rueffer A, Michalsen A, Dobos GJ. Noninvasive markers in the assessment of intestinal inflammation in inflammatory bowel diseases: performance of fecal lactoferrin, calprotectin, and PMN-elastase, CRP. and clinical indices. Am J Gastroenterol. (2008) 103:162-9. doi: 10.1111/j.1572-0241.2007.01556.x

27. Peyrin-Biroulet L, Reinisch W, Colombel JF, Mantzaris GJ, Kornbluth A, Diamond R, et al. Clinical disease activity, C-reactive protein normalisation and mucosal healing in Crohn's disease in the SONIC trial. Gut. (2014) 63:88-95. doi: 10.1136/gutjnl-2013-304984

28. Lahiff C, Safaie P, Awais A, Akbari M, Gashin L, Sheth S et al. The Crohn's disease activity index (CDAI) is similarly elevated in patients with Crohn's disease and in patients with irritable bowel syndrome. Aliment Pharmacol Ther. (2013) 37:786-94. doi: 10.1111/apt.12262
29. Frøslie KF, Jahnsen J, Moum BA, Vatn MH, IBSEN Group. Mucosal healing in inflammatory bowel disease: results from a Norwegian population-based cohort. Gastroenterology. (2007). 133:412-22. doi: 10.1053/j.gastro.2007.05.051

30. Ardizzone S, Cassinotti A, Duca P, Mazzali C, Penati C, Manes G et al. Mucosal healing predicts late outcomes after the first course of corticosteroids for newly diagnosed ulcerative colitis. Clin Gastroenterol Hepatol. (2011) 9:483-9.e3. doi: 10.1016/j.cgh.2010.12.028

31. Colombel JF, Rutgeerts P, Reinisch W, Esser D, Wang Y, Lang Y et al. Early mucosal healing with infliximab is associated with improved long-term clinical outcomes in ulcerative colitis. Gastroenterology. (2011) 141:1194201. doi: 10.1053/j.gastro.2011.06.054

32. Arai $M$, Naganuma $M$, Sugimoto $S$, Kiyohara $H$, Ono $K$, Mori $K$ et al. The ulcerative colitis endoscopic index of severity is useful to predict mediumto long-term prognosis in ulcerative colitis patients with clinical remission. $J$ Crohns Colitis. (2016) 10:1303-9. doi: 10.1093/ecco-jcc/jjw104

33. Boon GJ, Day AS, Mulder CJ, Gearry RB. Are faecal markers good indicators of mucosal healing in inflammatory bowel disease? World J Gastroenterol. (2015) 21:11469-80. doi: 10.3748/wjg.v21.i40.11469

34. Yamaguchi S, Takeuchi Y, Arai K, Fukuda K, Kuroki Y, Asonuma K et al. Fecal calprotectin is a clinically relevant biomarker of mucosal healing in patients with quiescent ulcerative colitis. J Gastroenterol Hepatol. (2016) 31:93-8. doi: 10.1111/jgh.13061

35. Kristensen V, Klepp P, Cvancarova M, Røseth A, Skar V, Moum B. Prediction of endoscopic disease activity in ulcerative colitis by two different assays for fecal calprotectin. J Crohns Colitis. (2015) 9:1649. doi: 10.1093/ecco-jcc/jju015

36. Naismith GD, Smith LA, Barry SJ, Munro JI, Laird S, Rankin K et al. A prospective single-centre evaluation of the intraindividual variability of faecal calprotectin in quiescent Crohn's disease. Aliment Pharmacol Ther. (2013) 37:613-21. doi: 10.1111/apt.12221

Conflict of Interest: The content of this manuscript has been presented in part at the IBD 2017-Therapeutic and Biological Barriers at Symposium 209 (Berlin) in October 2017.

The authors declare that the research was conducted in the absence of any commercial or financial relationships that could be construed as a potential conflict of interest.

Publisher's Note: All claims expressed in this article are solely those of the authors and do not necessarily represent those of their affiliated organizations, or those of the publisher, the editors and the reviewers. Any product that may be evaluated in this article, or claim that may be made by its manufacturer, is not guaranteed or endorsed by the publisher.

Copyright $\odot 2021$ Chen, Hu, Fan and Lv. This is an open-access article distributed under the terms of the Creative Commons Attribution License (CC BY). The use, distribution or reproduction in other forums is permitted, provided the original author(s) and the copyright owner(s) are credited and that the original publication in this journal is cited, in accordance with accepted academic practice. No use, distribution or reproduction is permitted which does not comply with these terms. 